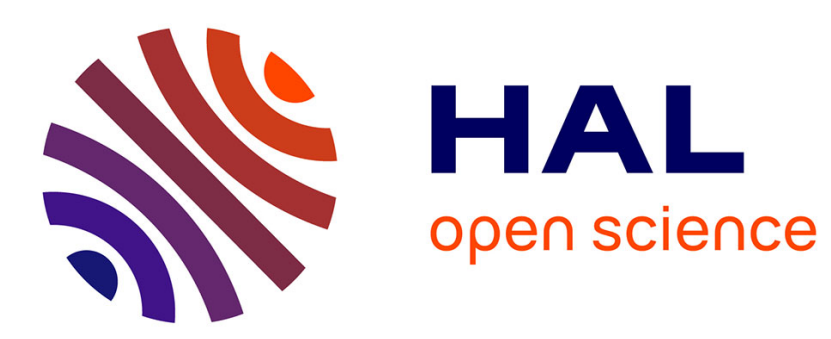

\title{
A GLOBAL TIME DOMAIN CIRCUIT SIMULATION OF A MICROWAVE RECTENNA
}

\author{
Brahim Essakhi, Gilles Akoun, Lionel Pichon
}

\section{To cite this version:}

Brahim Essakhi, Gilles Akoun, Lionel Pichon. A GLOBAL TIME DOMAIN CIRCUIT SIMULATION OF A MICROWAVE RECTENNA. International Journal of Numerical Modelling: Electronic Networks, Devices and Fields, 2007, 6th International Workshop on Computational Electromagnetics in the Time-Domain (CEM-TD), 20 (1-2), pp.3-15. 10.1002/jnm.626 . hal-02985025

\section{HAL Id: hal-02985025 \\ https://hal.science/hal-02985025}

Submitted on 1 Nov 2020

HAL is a multi-disciplinary open access archive for the deposit and dissemination of scientific research documents, whether they are published or not. The documents may come from teaching and research institutions in France or abroad, or from public or private research centers.
L'archive ouverte pluridisciplinaire HAL, est destinée au dépôt et à la diffusion de documents scientifiques de niveau recherche, publiés ou non, émanant des établissements d'enseignement et de recherche français ou étrangers, des laboratoires publics ou privés. 


\title{
A GLOBAL TIME DOMAIN CIRCUIT SIMULATION OF A MICROWAVE RECTENNA
}

\author{
Brahim Essakhi $^{1}$, Gilles Akoun ${ }^{2}$, Lionel Pichon ${ }^{1}$ \\ ${ }^{1}$ LGEP, UMRS 8507 CNRS, SUPELEC, \\ UNIVERSITE PARIS SUD, UNIVERSITE PIERRE ET MARIE CURIE \\ Plateau du Moulon, 91192 Gif-sur-Yvette, France \\ ${ }^{2}$ EADS CCR - 12 rue Pasteur, 92150 Suresnes, France
}

\section{Introduction}

Wireless power transfer is a promising technique for low-power applications: signal detection, wireless control systems, radio-frequency identification (RFID) applications etc. The key component of this transfer is the rectenna: the rectenna combines a rectifying circuit and an antenna. The antenna receives the microwave power and the rectifying circuit converts it into electric power.

Power transmitting and receiving systems must be designed so that the power transmitted from the transmitting antenna is transmitted efficiently to the rectenna and is converted into DC power by rectifiers. The design and characterization of a rectenna requires a global analysis taking into account both electromagnetic modelling (for distributed parts) and circuit simulation (for lumped components). In [1] the performances of a rectenna were predicted using a combination of a method of moment for the passive part and a harmonic balance simulation non-linear circuit analysis for the rectification process.

In this work a circuit model allowing a global simulation of a rectenna structure is presented. This model takes into account both distributed electromagnetic parts of the antenna and the rectifier circuit. It provides a straightforward way to determine the power transmitted to the load, when the antenna is illuminated by a transient incident wave. The model is built in two steps. In a first step an equivalent circuit of the antenna, when illuminated by an incident field, is determined this is performed by a 3D finite element model in the time domain. The impedance is computed over a wide frequency band and an accurate rational approximation is obtained from the technique proposed in [2]. The electromotive force (emf) induced by the transient excitation is computed from a 3D finite element time domain scattering problem. In a second step the equivalent circuit of the antenna and the lumped elements of the rectifying circuit are incorporated into a circuit simulator. The resulting circuit provides a reliable nonlinear time domain circuit simulation of the whole structure with PSPICE from which the energy transmitted to the load can be deduced.

\section{STUDIED RECTENNA}

The studied rectenna allowing RF-to-DC conversion is shown in Fig.1. It consists of a receiving loop antenna and a rectifier circuit feeding a passive load. The dimensions of the loop antenna are presented in Fig.2. The thickness of the conductor (a few ten micrometers is supposed to be negligible). The loop antenna is assumed perfectly conducting. 


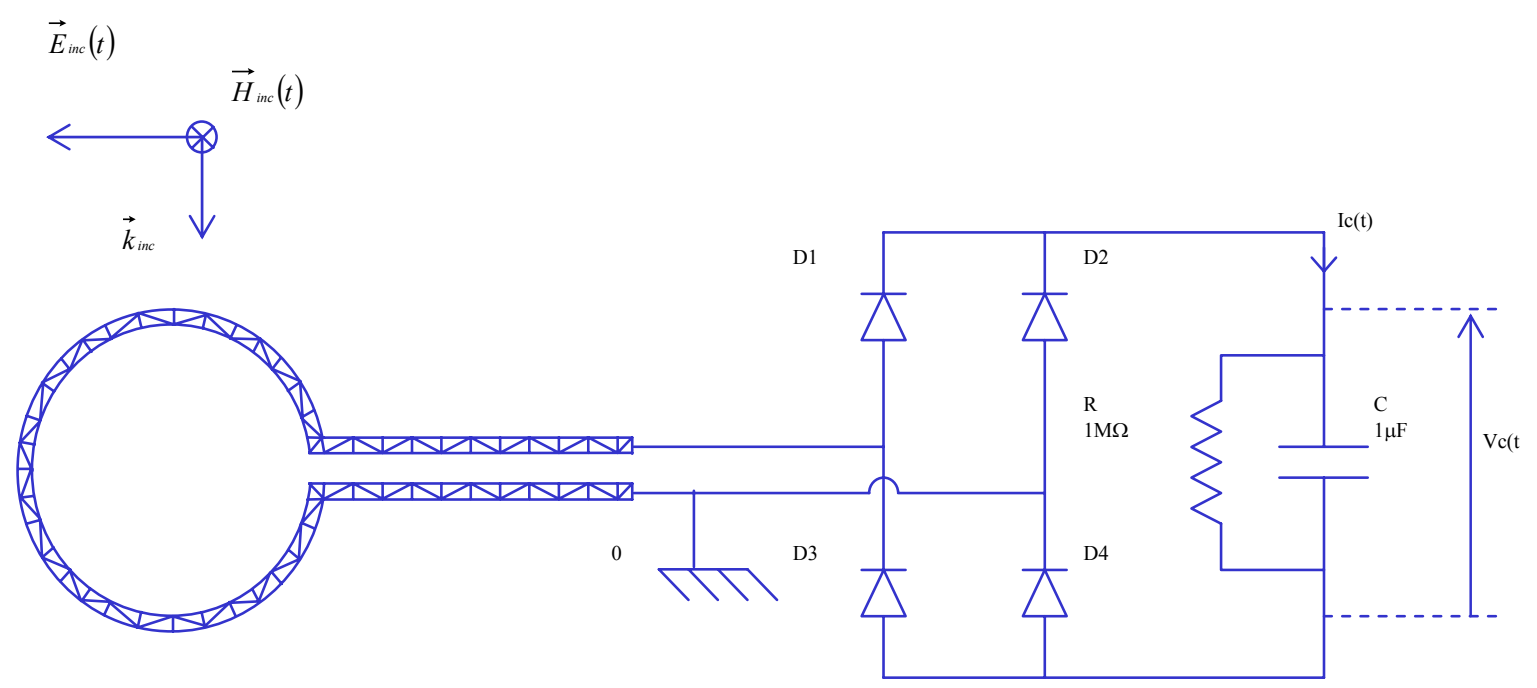

Fig. 1: Rectenna with RF-to-DC circuit.

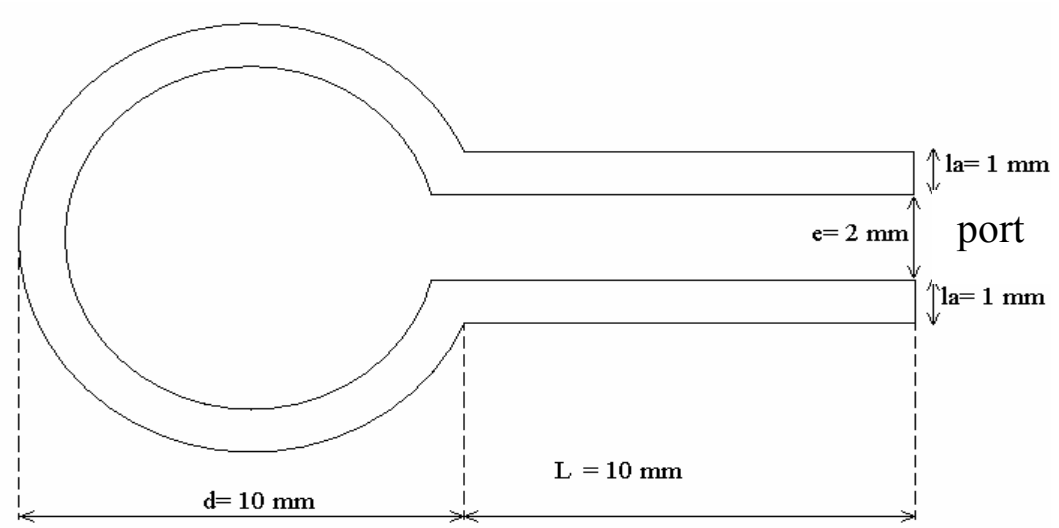

Fig.2: Dimensions of the loop antenna.

\section{ELECTROMAGNETIC ANALYSIS}

\section{Finite element method in time domain}

The electromagnetic analysis of the loop antenna is achieved by a finite element time domain approach. In the time domain, the electromagnetic problem is described with the double curl's equation given by:

$$
\nabla \times \frac{1}{\mu} \nabla \times \mathrm{E}+\sigma \partial_{t} \mathrm{E}+\varepsilon \partial_{t^{2}}^{2} \mathrm{E}=-\partial_{t} J_{i m p}
$$

We consider the Hilbert space $\left(H_{\text {curl }}(\Omega),\langle., .\rangle_{\Omega}\right)$ as a space of the work, where $\Omega$ is the volume of the computation. By multiplying Eq.(1) by a vector testing function $F \in H_{\text {curl }}(\Omega)$, integrating on the considered computational volume and using Green's first identity, we perform the variational formulation of the electromagnetic problem, given by: 


$$
\begin{array}{r}
\left\langle\frac{1}{\mu} \nabla \times E, \nabla \times F\right\rangle_{\Omega}+\left\langle\sigma \partial_{t} E, F\right\rangle_{\Omega}+\left\langle\varepsilon \partial_{t^{2}}^{2} E, F\right\rangle_{\Omega}+ \\
\left\langle\frac{1}{\mu} n \times \nabla \times E, F\right\rangle_{\partial \Omega}=-\left\langle\partial_{t} J_{\text {imp }}, F\right\rangle_{\Omega}, \forall F \in H_{\text {curl }}(\Omega)
\end{array}
$$

where $\partial \Omega$ is the boundary surface, $n$ is the unit outward field vector normal to $\partial \Omega$.

\section{Edge element interpolation}

We discretize the space domain with tetrahedral elements and write the electric vector in terms of Whitney basis functions associated with the edges of these elements $[3,4]$.

$$
E(r, t)=\sum_{a \in A} v_{a}(t) W_{a}(r)
$$

where $A$ is all the edges of the mesh, $W_{a}$ is the vector basis function associated with the edge $a$ and $v_{a}$ corresponds to the circulation of the electric field along the edge $a$. Fig.3 shows a typical mesh used in the computation.

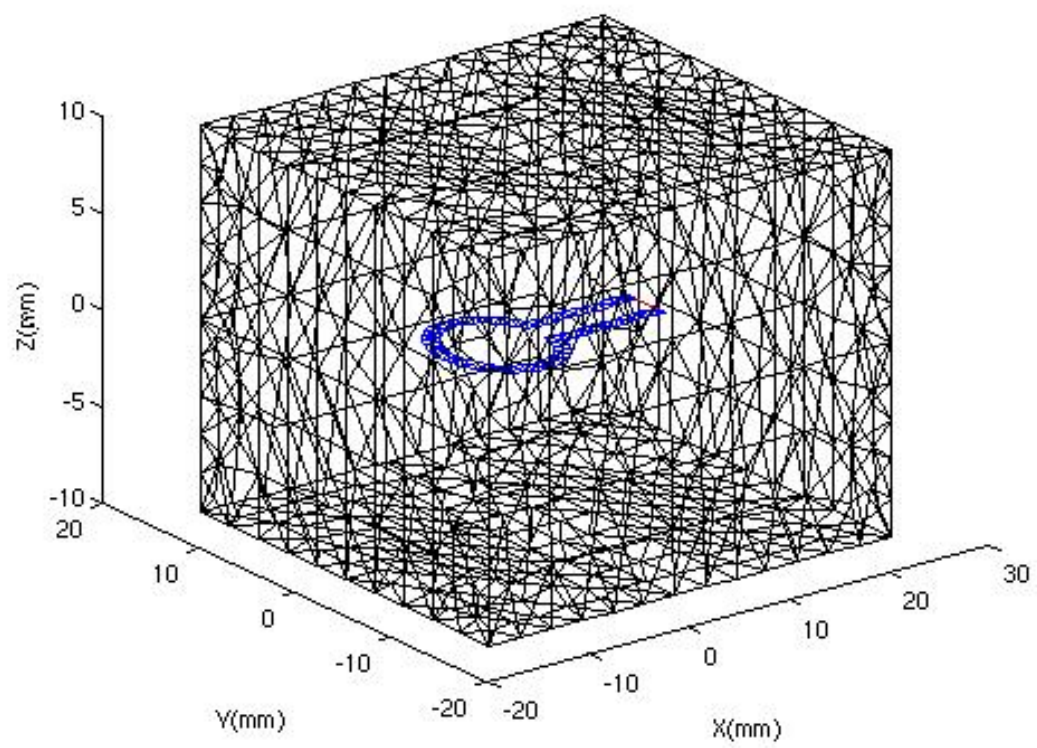

Fig.3: Computational domain.

\section{Truncation of the unbounded domain}

The domain in which we compute the fields must be bounded in order to perform the finite element solutions. We have chosen to use Silver-Müller condition (in first order), because it is easy to implement and allows to obtain a satisfactory results [5]. It is given by:

$$
n \times \nabla \times E=\frac{1}{c} \partial_{t} E_{\tan }
$$


where $c$ is the velocity of light and $E_{\tan }$ is the tangential electric field at the outer boundary surface.

\section{Global finite element system}

From Eqs.(2), (3), (4) and using the Galerkin method ( $\left.F=W_{b}\right)$ putting the test function as basis function, we have:

$$
\mathbf{M}_{m} \ddot{\mathbf{v}}(t)+\mathbf{M}_{a} \dot{\mathbf{v}}(t)+\mathbf{M}_{r} \mathbf{v}(t)=L(t),
$$

where $\mathbf{v}$ is the vector of unknowns, $L$ is the vector of the excitation currents and $\mathbf{M}_{m}, \mathbf{M}_{r}$ and $\mathbf{M}_{\sigma}$ are time-independent matrices. The elementary terms of these matrices are given by:

$$
\begin{aligned}
& \left(\mathbf{M}_{m}\right)_{a b}=\left\langle\varepsilon W_{a}, W_{b}\right\rangle_{\Omega}, \\
& \left(\mathbf{M}_{r}\right)_{a b}=\left\langle\frac{1}{\mu} \nabla \times W_{a}, \nabla \times W_{b}\right\rangle_{\Omega}, \\
& \left(\mathbf{M}_{\sigma}\right)_{a b}=\left\langle\sigma W_{a}, W_{b}\right\rangle_{\Omega}+\left\langle\sqrt{\frac{\varepsilon}{\mu}} W_{\tan , a}, W_{\tan , b}\right\rangle_{\Gamma_{A B C}}, \\
& L_{a}=-\left\langle W_{a}, \partial_{t} J_{i m p}\right\rangle_{\Omega},
\end{aligned}
$$

where $\Gamma_{A B C}$ represents the surface where the Silver-Müler condition is imposed.

\section{The Newmark scheme}

In order to solve the ODE Eq.(5) step by step in time, the time derivatives must be approximated by finites differences. We use the Newmark method given by:

$$
\begin{aligned}
\mathbf{v}_{n+1} & =\mathbf{v}_{n}+\delta t \dot{\mathbf{v}}_{n}+0.5 \delta t^{2}\left[2 \beta \ddot{\mathbf{v}}_{n+1}+(1-2 \beta) \ddot{\mathbf{v}}_{n}\right], \\
\dot{\mathbf{v}}_{n+1} & =\dot{\mathbf{v}}_{n}+\delta t\left[\gamma \ddot{\mathbf{v}}_{n+1}+(1-\gamma) \ddot{\mathbf{v}}_{n}\right],
\end{aligned}
$$

characterized by two parameters $\beta$ and $\gamma$ which control the stability of the accuracy. This scheme is unconditionally stable if the two parameters verify: $2 \beta \geq \gamma \geq \frac{1}{2}[5,6]$. The problem to solve at each time step is given by:

$$
\begin{array}{r}
{\left[\mathbf{M}_{m}+\gamma \delta t \mathbf{M}_{a b s}+\beta \mathbf{M}_{r}\right] \mathbf{v}_{n+1}=\left[2 \mathbf{M}_{m}-(1-2 \gamma) \delta t \mathbf{M}_{a b s}-\left(\frac{1}{2}-2 \beta+\gamma\right) \delta t^{2} \mathbf{M}_{r}\right] \mathbf{v}_{n}+} \\
{\left[-\mathbf{M}_{m}+(\gamma-1) \delta t \mathbf{M}_{a b s}-\left(\frac{1}{2}+\beta-\gamma\right) \delta t^{2} \mathbf{M}_{r}\right] \mathbf{v}_{n-1}+\left[\beta \mathbf{L}_{n+1}+(1-2 \beta) \mathbf{L}_{n}+\beta \mathbf{L}_{n-1}\right] \delta t^{2}}
\end{array}
$$

If we choose $2 \beta=\gamma=\frac{1}{2}$, this scheme have a second-order accuracy. 
We solve an equation of the form $\mathbf{A x}=\mathbf{b}$ in each time step, where $\mathbf{A}$ is a sparse matrix.

\section{EQUIVALENT CIRCUIT OF THE ANTENNA}

To obtain an equivalent circuit of the rectenna under PSPICE, we have to achieve an equivalent circuit of the receiving loop antenna when it is illuminated by an incident electromagnetic field. Both the impedance of the antenna over the whole frequency band ]0,20] $\mathrm{GHz}$ and the electromotive force induced have to be determined.

\section{The impedance of the antenna}

The antenna is excited by a time varying impressed current between the ends of the two arms called the port (Fig.2) given by $i(t)=1 \sin (\omega t)$. The voltage is obtained by solving Eq.(9). As an example and for a given frequency in $f_{0}=2 \mathrm{GHz}$, Fig.4 shows the prescribed current and the computed voltage on the port. As expected, the voltage reaches a sinusoidal steady state. The resistance $\left(R_{m}\right)$ of the antenna and the reactance $\left(X_{m}\right)$ are given by Eq. $(11)$, where $I_{\max }$ is the peak current, $P_{a v}$ and $Q_{a v}$ are respectively the active and reactive power, and $i_{q}(t)=i\left(t-\frac{\pi}{2}\right)$.

$$
\begin{aligned}
& P_{a v}=\frac{1}{2} R_{m} I_{\max }{ }^{2}=\frac{1}{T} \int_{T} v(t) \cdot i(t) d t, \\
& Q_{a v}=-\frac{1}{2} X_{m} I_{\max }^{2}=\frac{1}{T} \int_{T} v(t) i_{q}(t) d t
\end{aligned}
$$

The impedance of the loop antenna is deduced:

$$
Z=R_{m}+j X_{m}
$$

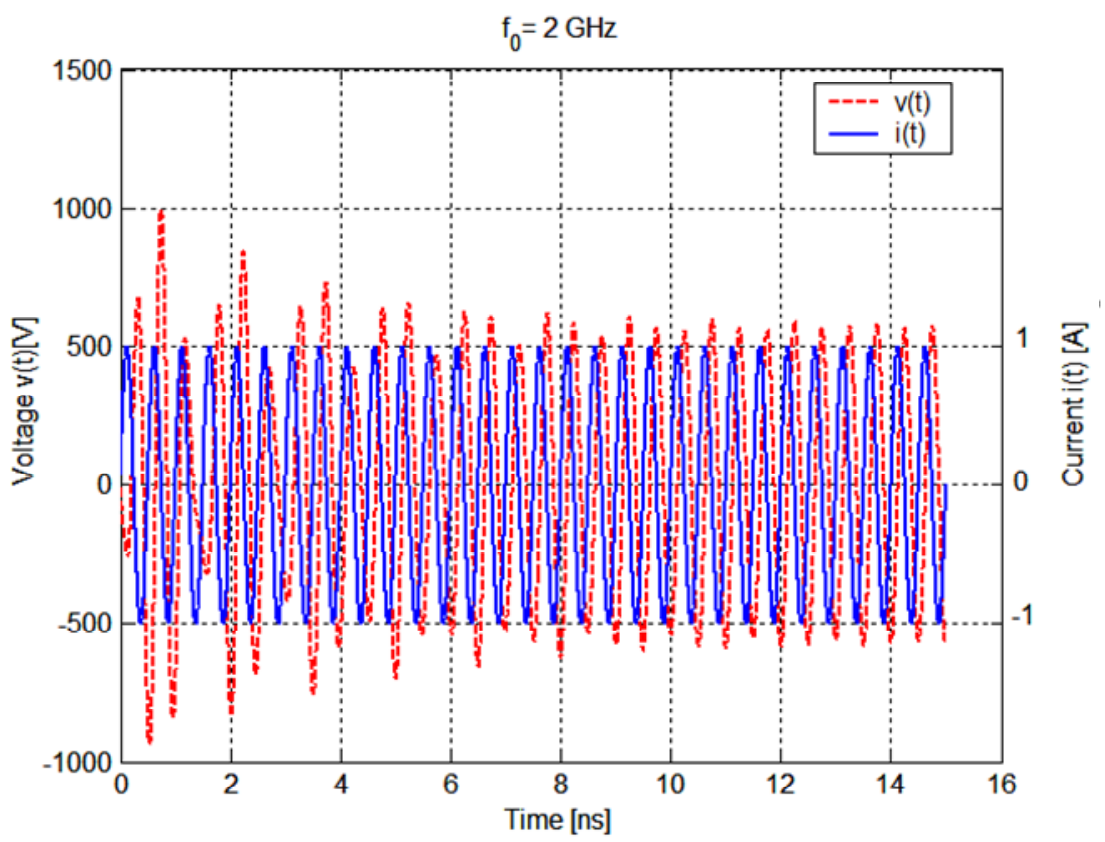

Fig.4: Impressed current $i(t)$ and obtained voltage $v(t)$ on the port. 
The same procedure is achieved for 60 points in ]0,20] $\mathrm{GHz}$ in order to characterize the antenna over the whole frequency band. Fig.5 and Fig. 6 show the comparison between the results using this approach and two other frequency domain approaches: the BEM approach used by EADS CCR team and a standard frequency domain FEM by using Ansys software. The curves underline the same behaviour over the studied band. In particular, the resonances peaks obtained are in reasonable agreement with the EADS CCR's curves. The main error sources may be due to poor spatial discretization around the port in this example. Moreover improved boundary conditions may provide more accurate results. Since in the electromagnetic analysis first order edge elements are used, second order boundary conditions (or higher order) involving tangential derivatives cannot be prescribed. The best solution would be to develop PML (Perfectly Matched Layers) which can be easily combined with first order elements [7]. In any case the additionnal CPU time involved by the boundary condition is negligible when compared to the whole CPU time resulting from the $3 \mathrm{D}$ finite element analysis.

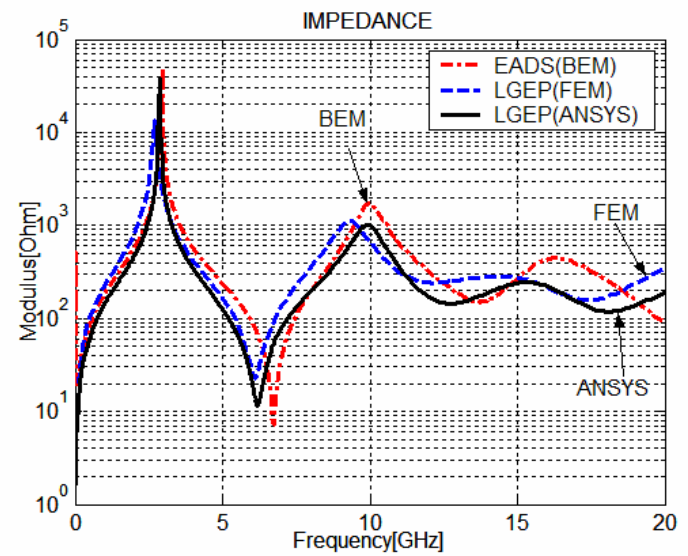

Fig.5: Modulus of the impedance.

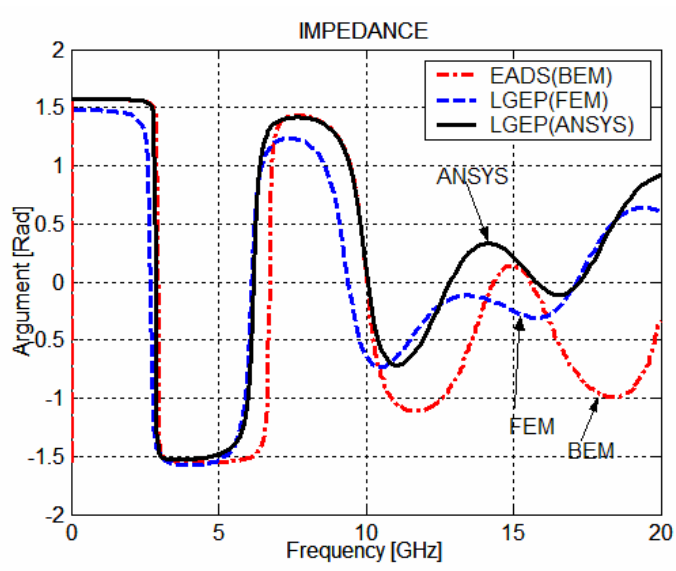

Fig.6: Argument of the impedance.

In order to take account of this impedance under PSPICE we have to perform a modal decomposition of the impedance $Z$ over the broad frequency band. An approximate expression $Z^{k}$ of $Z$ is searched by dividing the whole frequency band $\Omega$ into $N_{b}$ intervals $\Omega_{k}\left(1 \leq k \leq N_{b}\right)$ corresponding to the resonance peaks. Over each $\Omega_{k}$ we approximate the impedance by $Z^{k}$, hence:

$$
Z(s)=\sum_{k=1}^{N_{b}} \frac{\sum_{j=0}^{N_{k}} a_{j}^{k} s^{j}}{\sum_{i=0}^{D_{k}} b_{i}^{k} s^{i}},
$$

On the band $\Omega_{k}$, Eq.(13) is written for a series of $M_{k}$ values $s_{l}$ in order to compute the coefficients of rational approximation over each interval. The technical details about this approximation can be found in a previous paper [2]. 


\section{The electromotive force of the antenna}

As a test case the antenna is illuminated by a transient plane wave whose electric field is shown in Fig.7. Before $5 \mathrm{~ns}$ the excitation signal is a sinusoidal source with frequency $f=1 \mathrm{GHz}$. At $5 \mathrm{~ns}$ the source becomes zero.

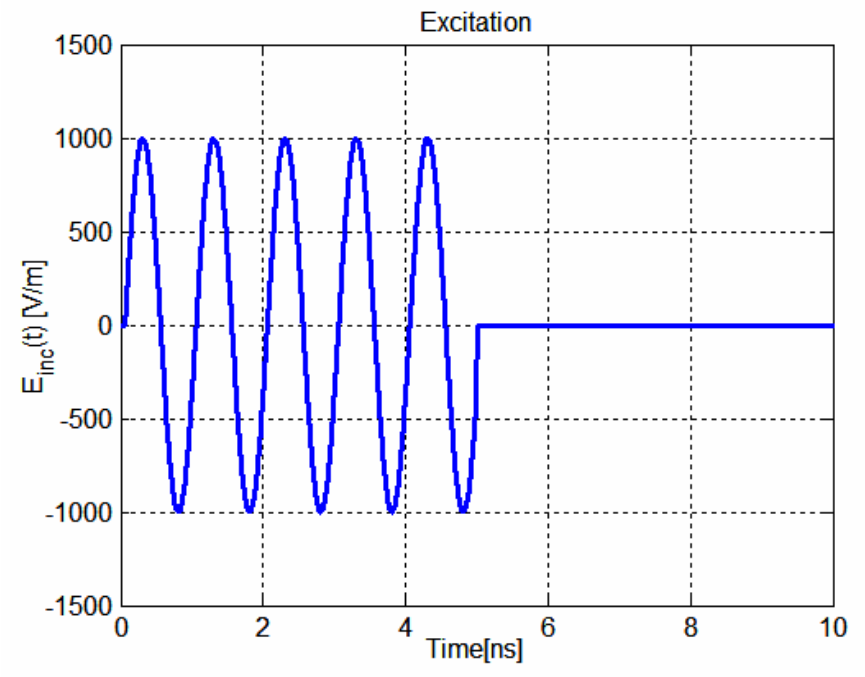

Fig.7: The incident electric field over 5 periods.

The problem is solved in terms of the scattered electric field:

$$
\left\{\begin{array}{l}
\nabla \times \frac{1}{\mu} \nabla \times E^{\text {scat }}+\sigma \partial_{t} E^{\text {scat }}+\varepsilon \partial_{t t} E^{\text {scat }}=0, \text { in } \Omega \\
n \times E^{\text {scat }}=-n \times E^{i n c}, \text { on the antenna } \\
n \times \nabla \times E^{\text {scat }}=\frac{1}{c_{0}} \partial_{t} E_{\mathrm{tan}}^{\text {scat }}, \text { on } \partial \Omega
\end{array}\right.
$$

Fig. 8 shows the induced voltage $V(t)$ on the port of the antenna.

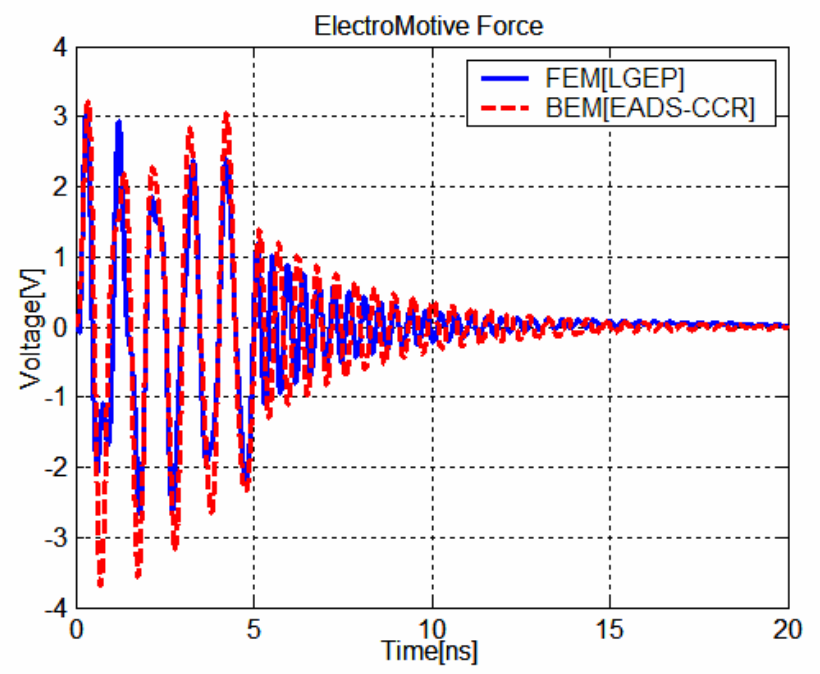

Fig.8: The output voltage on the port of the antenna. 
Fig. 8 shows the comparison between the FEM results, and the voltage using the BEM method from EADS (after an Inverse Fourier Transform). The two curves underline the same behaviour. It is worth noting that $V(t)$ first varies according to the exciting frequency of 1 $\mathrm{GHz}$; when the excitation signal becomes zero at $5 \mathrm{~ns}$, the voltage $V(t)$ oscillates around the frequency corresponding to the first resonance peak $(3 \mathrm{GHz})$ of the antenna (Fig.8). The distribution of the time variation of the electric field on the plane containing the antenna is given after $5 \mathrm{~ns}$ (when the excitation source is zero).

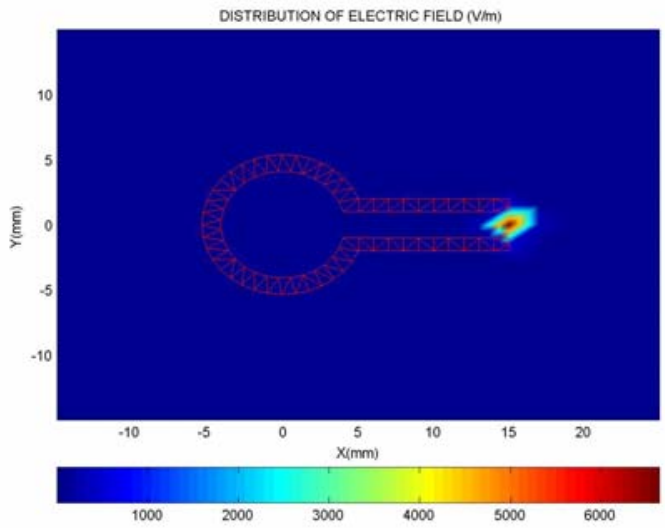

$$
t=5.005 \mathrm{~ns}
$$

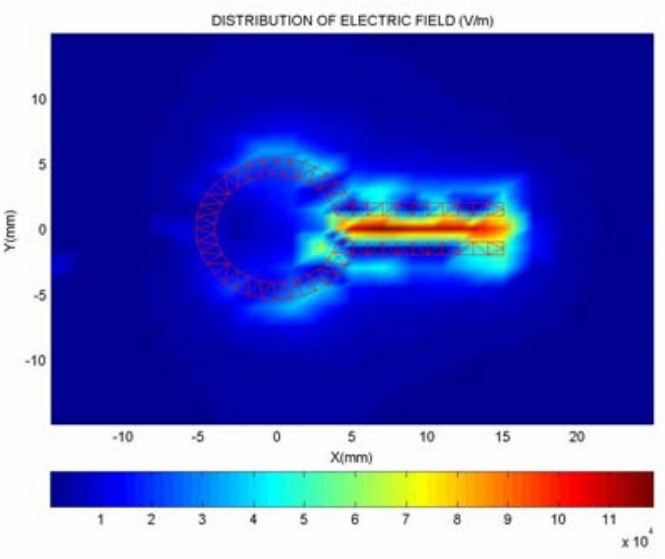

$$
t=5.135 \mathrm{~ns}
$$

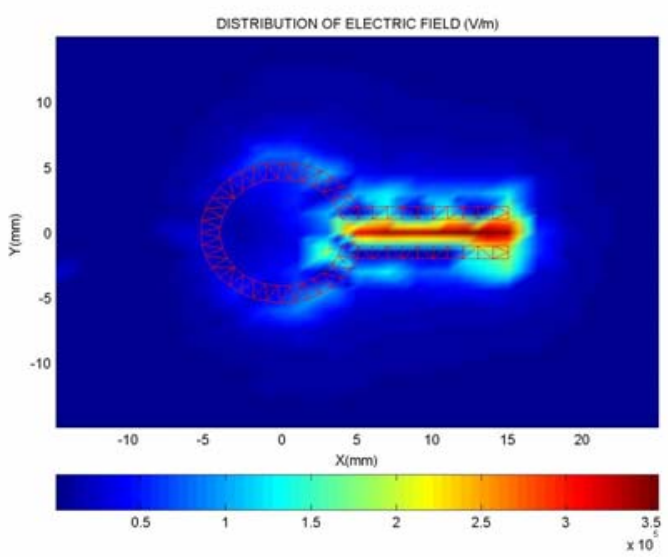

$$
t=5.265 \mathrm{~ns}
$$

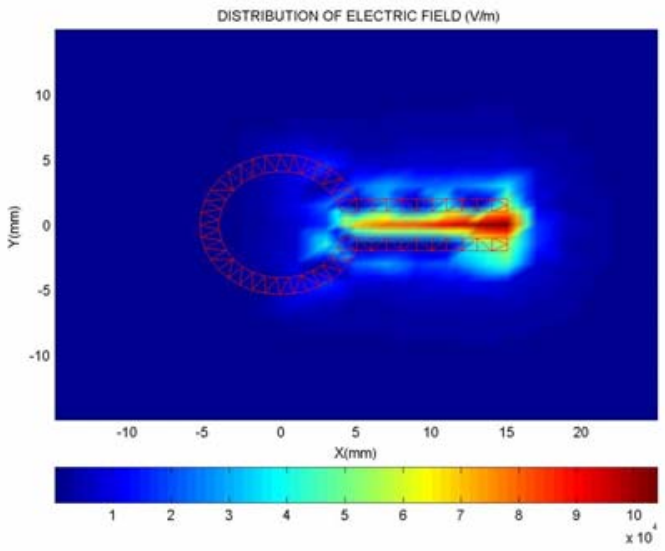

$t=5.070 \mathrm{~ns}$

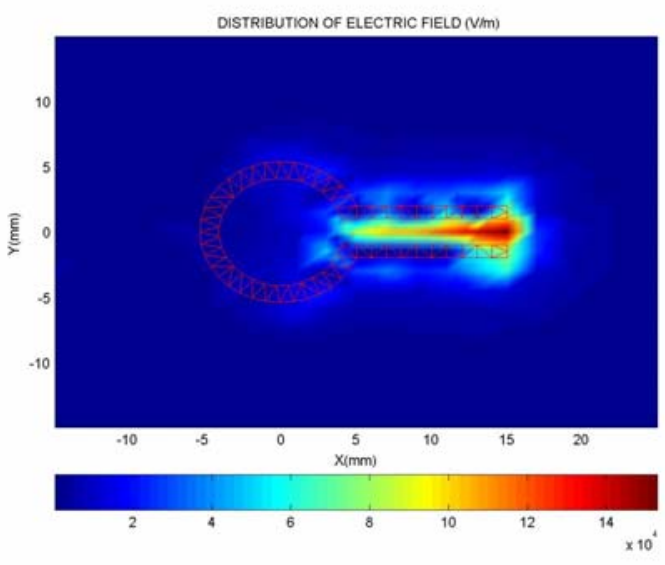

$t=5.200 \mathrm{~ns}$

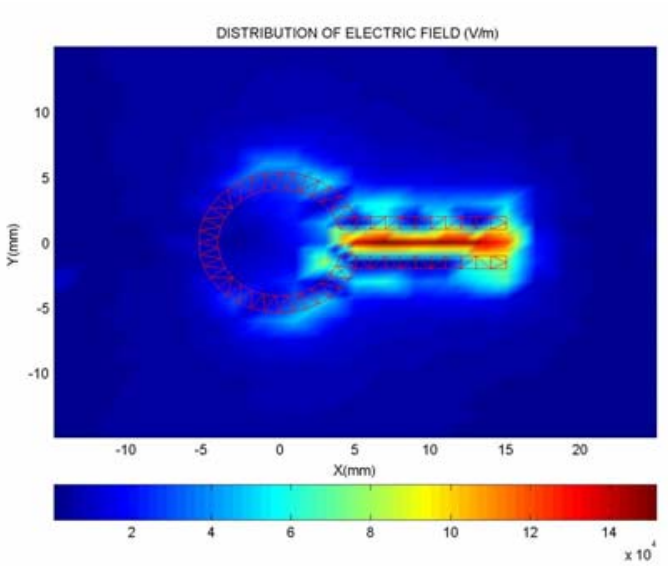

$t=5.333 n s$

Fig. 9: Distribution of the electric field over the plane of the antenna (after $5 \mathrm{~ns}$ ). 
From the previous study, the equivalent circuit of the antenna when illuminated by the incident field is shown in Fig.10.

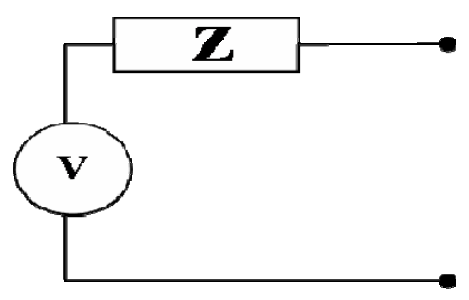

Fig.10: The equivalent circuit of the loop antenna.

\section{TIME DOMAIN CIRCUIT ANALYSIS}

Once the equivalent circuit of the antenna is known, a global simulation of the rectenna under an electronic simulator is possible. In this study the impedance of the loop antenna was taken into account with 3 order rational functions. The corresponding PSPICE circuit blocks are shown in Fig.11.

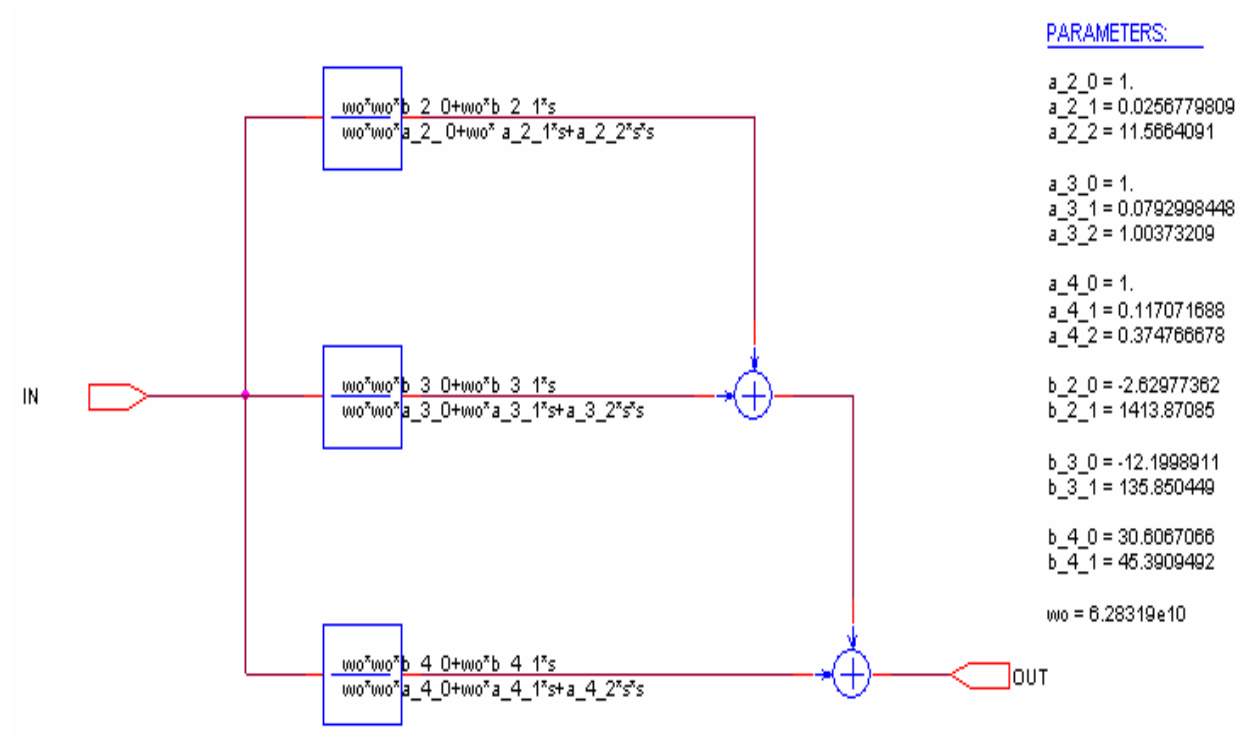

Fig. 11: PSPICE schematics of the impedance of the loop antenna (Z_11 in Fig.12).

Fig.12 shows the whole equivalent circuit of the rectenna under PSPICE. It includes the impedance of the antenna $\left(Z=Z_{-} 11\right)$ and the emf $(V=V 2)$ computed above, the non-linear components (diodes) and the load. 


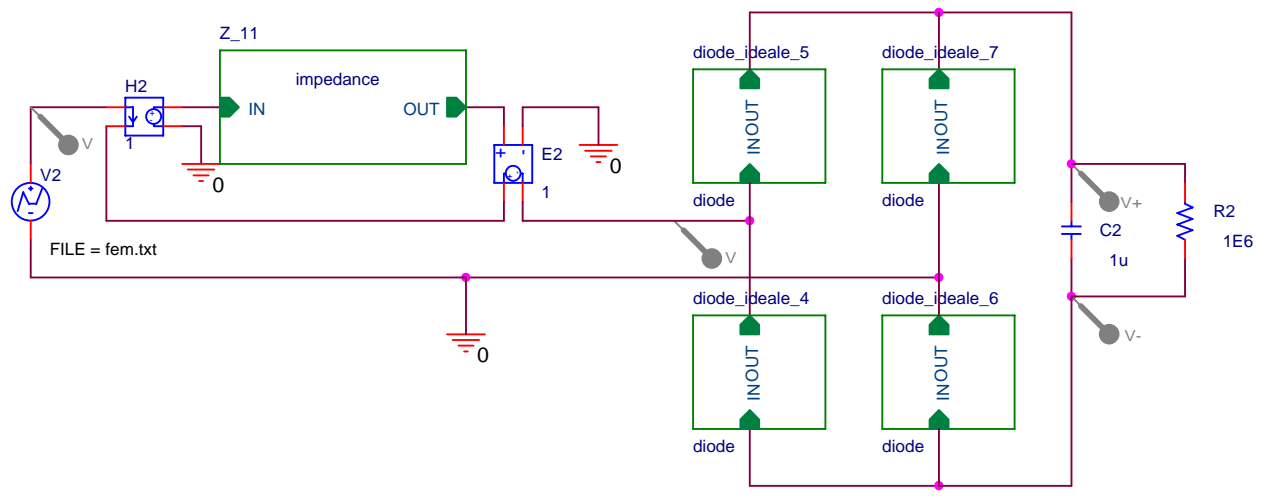

Fig. 12: The equivalent circuit of the rectenna under PSPICE.

Using this circuit under an electronic simulator we can compute the output voltage the time domain variation of $V_{C}(t)$ on the capacitor when the antenna is illuminated by the transient excitation source described in Fig.7. Fig.13 shows this voltage using PSPICE. Results are in a good agreement with those obtained from Saber. The energy stored in the capacitor can be deduced:

$$
\left.\mathrm{W}_{\text {ess }}(\mathrm{t})=\frac{1}{2} \mathrm{CV}_{\mathrm{c}}^{2} \mathrm{t}\right)
$$

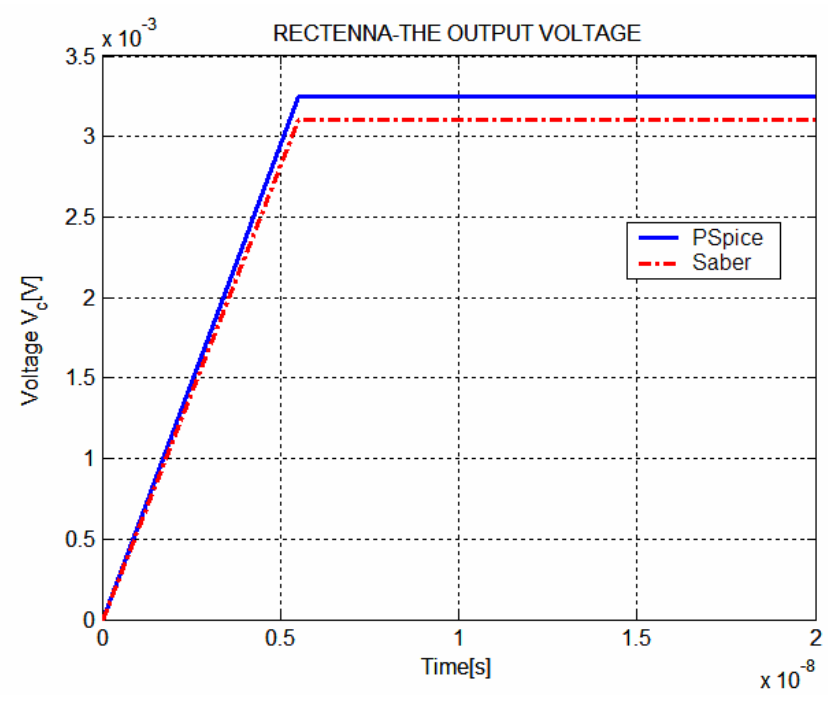

Fig.13: The output voltage $V_{C}(t)$ on the capacitor.

\section{Conclusion}

We showed in this paper how an efficient global circuit simulation of a microwave rectenna can be performed. From a 3D Finite Element Time Domain model an equivalent circuit of the antenna illuminated by an incident wave was determined: the impedance was computed over a wide frequency band and the induced electromotive force was computed when solving a transient scattering problem. This resulting equivalent circuit of the antenna has been incorporated into the electronic simulator PSPICE, together with the lumped components of the rectenna (diodes and load). Thus a global non-linear time domain analysis 
of the whole structure is available. Such an approach could improve the design stage of rectenna devoted to microwave power transfer.

The technique presented in this paper deals with both electromagnetic modeling and circuit simulation provides a first step towards the generation of behavioural models and macro-modelling of complex systems.

\section{References}

[1] Hagerty J.A., Popovic Z., An experimental and theoretical characterization of a broadband arbitrarily-polarized rectenna array, IEEE MTT-S International Microwave Symposium, Piscataway, NJ, USA; 2001, (3):1855-1858.

[2] Leroy P., Akoun G., Essakhi B., Santandrea L., Pichon L., An efficient global analysis of a rectenna using the combination of full-wave model and a rational approximation, 2005,. Eur. Phys. J. Appl. Phys. 29, 39-43.

[3] Bossavit A. A rational for edge-elements in 3D filed computations. IEEE Transactions on Magnetics 1988, M-24(1):74-79.

[4] Web JP. Edge elements and what they can do for you. IEEE Transactions on Magnetics 1993; M-25(2):1460-1465.

[5] Carpes W.P. Jr, Pichon L., Razek A., A finite element method for the numerical modelling of bounded and unbounded electromagnetic problems in the time domain, International Journal of Numerical Modelling, 2000, vol.13, pp 527-540.

[6] Gedney S, Navsariwala U, An unconditionally stable finite element time-solution of the vector wave equation, IEEE Microwave Guided Wave Letters 1995; 5, (10):332-334.

[7] Sacks Z., Kingsland D.M, Lee R., Lee J-F., A perfectly matched anisotropic absorber for use as an absorbing boundary condition, IEEE Transactions on Antennas and Propagation 1995; 43, (12):1460-1463. 